\title{
Contributions of alveolar epithelial cell quality control to pulmonary fibrosis
}

\author{
Jeremy Katzen ${ }^{1}$ and Michael F. Beers ${ }^{1,2}$ \\ 'Pulmonary, Allergy, and Critical Care Division, Department of Medicine, and Penn-CHOP Lung Biology Institute, Perelman School of Medicine at the University of Pennsylvania, Philadelphia, Pennsylvania, USA.
}

\begin{abstract}
Epithelial cell dysfunction has emerged as a central component of the pathophysiology of diffuse parenchymal diseases including idiopathic pulmonary fibrosis (IPF). Alveolar type 2 (AT2) cells represent a metabolically active lung cell population important for surfactant biosynthesis and alveolar homeostasis. AT2 cells and other distal lung epithelia, like all eukaryotic cells, contain an elegant quality control network to respond to intrinsic metabolic and biosynthetic challenges imparted by mutant protein conformers, dysfunctional subcellular organelles, and dysregulated telomeres. Failed AT2 quality control components (the ubiquitin-proteasome system, unfolded protein response, macroautophagy, mitophagy, and telomere maintenance) result in diverse cellular endophenotypes and molecular signatures including ER stress, defective autophagy, mitochondrial dysfunction, apoptosis, inflammatory cell recruitment, profibrotic signaling, and altered progenitor function that ultimately converge to drive downstream fibrotic remodeling in the IPF lung. As this complex network becomes increasingly better understood, opportunities will emerge to identify targets and therapeutic strategies for IPF.
\end{abstract}

\section{Introduction}

Fibrosis is a common pathological manifestation of chronic disease in a variety of organ systems, including liver, kidney, and skin. In the distal lung, fibrotic remodeling represents a major complication of and contributor to the morbidity and mortality of several diffuse parenchymal lung diseases seen in clinical practice (1). The shared pathological derangement of these diseases is a disruption of normal bronchoalveolar architecture resulting in impaired oxygen diffusion from the alveolus to the capillary plexus with resultant hypoxemia. Fibrotic lung diseases make up the most common diagnosis at lung transplantation (2), and among them, the most pernicious subclass is idiopathic pulmonary fibrosis (IPF) (3).

Histopathologically, IPF is defined by the presence of the usual interstitial pneumonia (UIP) pattern, identified by the organization of myofibroblasts into fibroblastic foci in the context of temporally and spatially heterogeneous chronic interstitial fibrosis (4). While fibroblastic foci represent the cardinal lesions in UIP, microscopic honeycombing of distal lung regions is also seen wherein affected areas undergo bronchiolization, lined by both hyperplastic alveolar type 2 (AT2) cells and epithelia expressing a variety of more proximal airway cell markers, including those of basal, ciliated, and goblet cell lineages (5). An additional defining feature of UIP is hyperplasia of AT2 cells and loss of alveolar type 1 (AT1) cells, suggesting that the pathological hallmarks of IPF are as much defined by aberrant epithelial cells as by mesenchymal expansion (6).

Two antifibrotic medications, nintedanib and pirfenidone, are approved for the treatment of IPF. While each attenuates the rate of lung function decline, neither halts progression or affects long-

Conflict of interest: The authors have declared that no conflict of interest exists. Copyright: ( 2020 , American Society for Clinical Investigation. Reference information: / Clin Invest. 2020;130(10):5088-5099. https://doi.org/10.1172/JCl139519. term survival (7-10). The refractoriness of the UIP lung to these therapies has led to a continued search for new therapeutic targets for IPF. In this Review, we focus on the biology of the dysfunctional alveolar epithelium in IPF, discuss a role for other epithelial lineages, and highlight the emerging importance of disrupted epithelial cell quality control as an upstream event in the cascade of aberrant injury/repair that results in the well-recognized clinical syndrome and end-stage pathology.

\section{The alveolar epithelium as a driver of IPF}

The role for a dysfunctional distal lung epithelium in IPF pathogenesis represents a rebirth of a concept first put forth by Haschek and Witschi in 1979, which was initially unsuccessful in challenging the contemporaneous view of lung fibrosis as an inflammatory disease (11). However, in part due to multiple setbacks experienced by clinical IPF trials based on pure antiinflammatory strategies, a refocused recognition of the distal lung epithelium as an active player in IPF emerged. Beginning in the early 2000s, the epithelial injury and abnormal wound repair model, postulating that recurrent microinjuries to a susceptible alveolar epithelium drive fibrotic remodeling, has become the dominant paradigm in IPF pathogenesis (Figure 1).

A central role for the AT2 cell in IPF is, in part, due to its critical function in alveolar niche homeostasis through the production of pulmonary surfactant (12) and as a progenitor cell to both self-renew and transdifferentiate into AT1 cells (13). Supporting this hypothesis are studies in murine models using diphtheria toxin or SR39TK herpes simplex virus- 1 thymidine kinase to ablate AT 2 cells. These studies established a dose-response effect that ranged from enhanced susceptibility to exogenous bleomycin-induced fibrosis with modest ablation, to spontaneous fibrosis following more substantive AT2 depletion (13-15). Subsequent studies showed that limitation of AT2 cell loss through pharmacological or genetic inhibition of apoptosis pathways also attenuated bleomycin fibrosis in mice (16). 


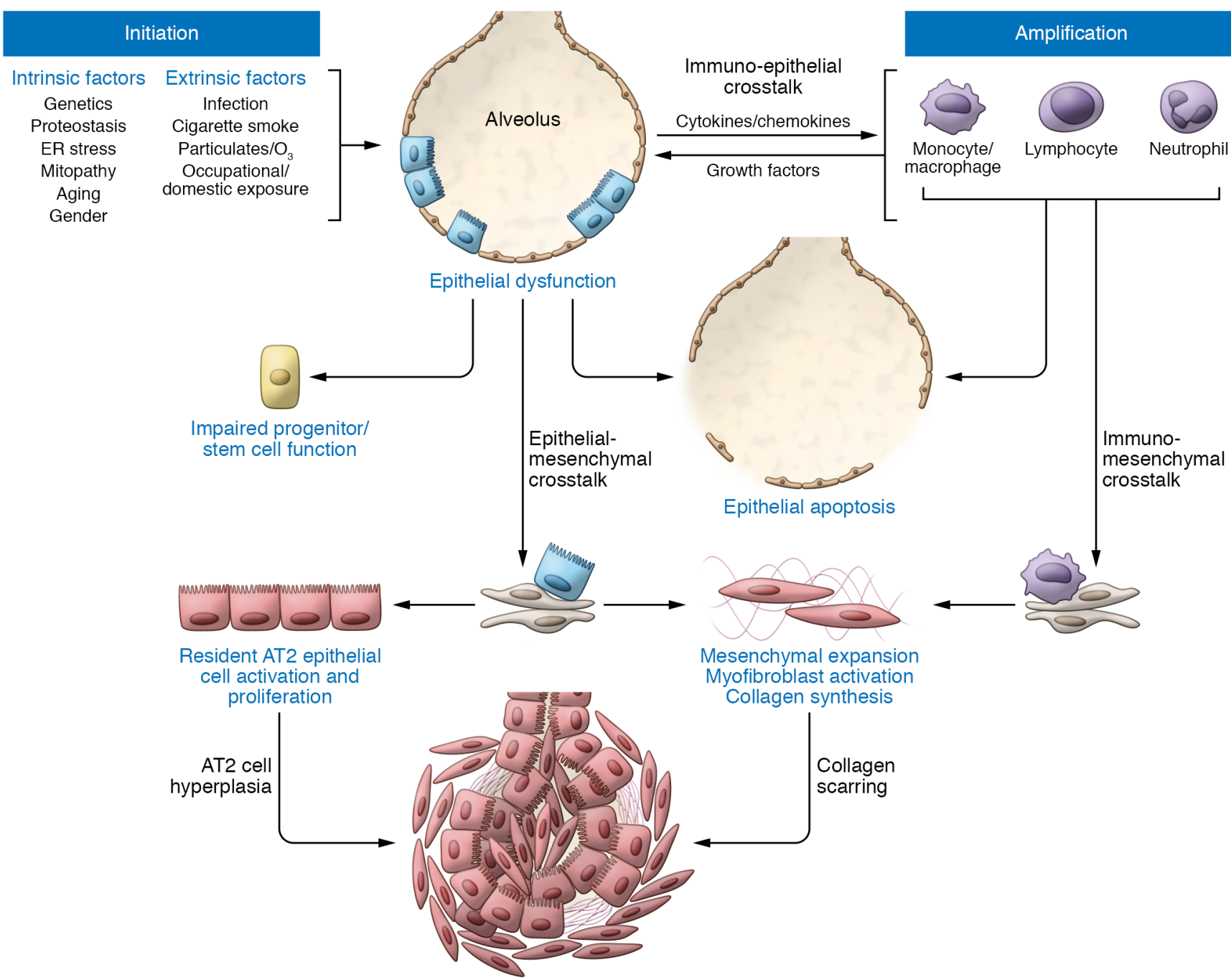

Fibrogenesis

Figure 1. IPF pathogenesis driven by epithelial dysfunction occurs in three phases. Initiation: Intrinsic (e.g., genetic) and extrinsic (e.g., infection, air pollution) factors acting through various pathways converge to produce a vulnerable alveolar type 2 epithelial cell (AT2) population (blue rectangular cells). Vulnerable AT2s subjected to continued intrinsic proteostatic/cell quality control challenges or additional secondary injurious stimuli (often recurrent) develop profound functional defects marked by aberrant activation of developmental programs, enhanced cell stress responses, impaired progenitor function, and/or apoptosis. Amplification: Dysfunctional AT2 cells (red rectangular cells) can initiate crosstalk with immune populations such as Ly6Chi monocytes, alveolar macrophages, neutrophils, or lymphocytes, which can both amplify the initial injury events and promote mesenchymal expansion further complemented by commensurate AT2/mesenchymal crosstalk. Fibrogenesis: The dysfunctional alveolar niche exhibits further feed-forward mechanisms to promote ongoing AT2 dysfunction marked by increased proliferation (AT2 hyperplasia), impaired transdifferentiation to AT1 cells, and upregulation of senescence programs. Coupled with enhanced myofibroblast activation and matrix deposition, the disrupted injury/ repair response leads to scar formation and progressive loss of lung architectural complexity culminating in progressive fibrotic remodeling, physiological derangements in gas exchange, and a clinically evident IPF phenotype.

These observations, coupled with the recognition that AT2 apoptosis is enhanced in human IPF $(17,18)$, have solidified a theory that disruption of alveolar epithelial homeostasis promotes lung fibrosis $(19,20)$. However, these studies did not address the mechanisms by which, beyond dropout, dysfunctional AT2 cells contribute to IPF development or progression.

\section{The AT2 cell and its quality control network}

AT2 cells face substantial biosynthetic and metabolic challenges as a consequence of dual roles: as progenitor cells as well as producers of surfactant phospholipids and proteins. The hardships imposed on AT2 cells by malformed proteins, accumulated macromolecules, dysfunctional mitochondria, and/or foreshortened telomeres have been implicated in a number of chronic lung diseases, including IPF (ref. 21 and Table 1). Like many eukaryotic cells, AT2 cells preserve homeostasis in the face of both endogenous challenges and a variety of exogenous insults to the fidelity of their biosynthetic, metabolic, and replicative machinery. AT2 cells have developed an integrative network of cell quality control repertoires (shown in Figure 2) to 


\section{Table 1. Dysfunctional cell quality control and lung disease}

\section{Cell quality control component Clinical diagnosis/phenotype Lung phenotype}

\section{Proteostasis}

SFTPC mutations

ChILD (children)
ILD (adult)
Epithelium-based molecular and cellular mechanism(s)

$N S I P \pm P A P$

UIP

Epithelial cytotoxicity from protein
aggregation or mistrafficking, including:
- UPR/ER stress
- Apoptosis
- Impaired autophagy
Cytokine/TCF- $\beta$ generation
Epithelial dysfunction:
- UPR/ER stress
- Necroptosis
Cytokine generation
UPR/ER stress
Impaired autophagy
ER stress
Impaired ubiquitin-proteasome system

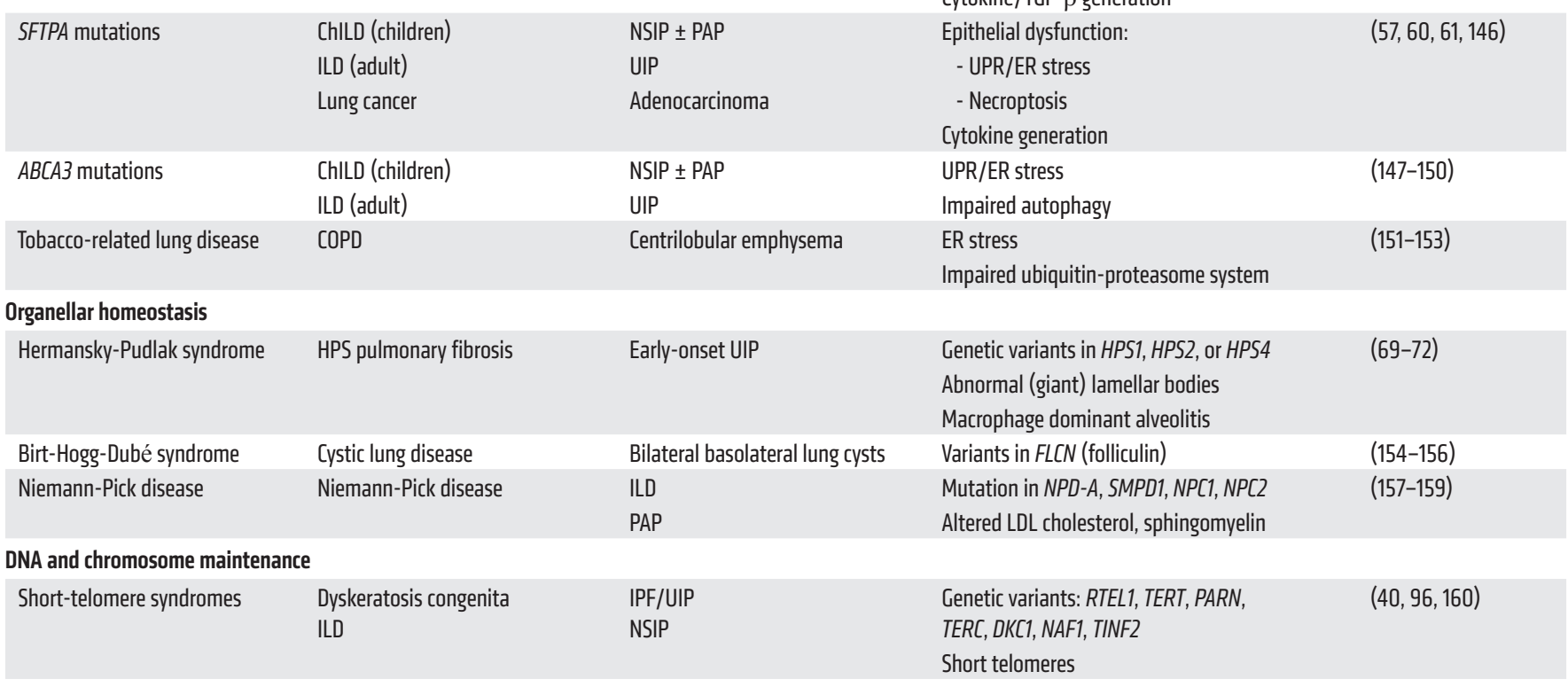

ChILD, interstitial lung disease of childhood; COPD, chronic obstructive pulmonary disease; ILD, interstitial lung disease; IPF, idiopathic pulmonary fibrosis; NSIP, nonspecific interstitial pneumonitis; PAP, pulmonary alveolar proteinosis; UIP, usual interstitial pneumonia.

manage, repair, and/or degrade damaged proteins, subcellular organelles, or chromosomes, including:

\section{Protein quality control: the proteostasis network}

Continuous mechanical or environmental stressors represent a constant threat to protein folding in the lung. Proteostasis refers to a network of processes handling protein folding, misfolding, unfolding, and degradation composed of a complex, dynamic, and interactive set of cellular systems and subcellular compartments (reviewed in refs. 21, 22). Disrupted proteostasis results in loss of function (e.g., insufficient active protein) and/or toxic gain of function (e.g., from aggregation). Of high relevance to chronic lung diseases like IPF is that the proteostatic capacity declines with age. Components of a prototypical proteostasis network response include some or all of the following:

Unfolded protein response. Misfolded conformers first activate three highly complementary unfolded protein response (UPR) signaling pathways that have evolved to ensure that ER protein folding capacity is not overwhelmed (reviewed in ref. 23). Specifically, distinct ER transmembrane sensors inositol-requiring enzyme 1 (IRE1), PKR-like endoplasmic reticulum kinase (PERK), and activating transcription factor 6 (ATF6) trigger one or more of three corresponding signaling cascades with two functional objectives: expanding ER refolding capacity and promoting translational attenuation. Cells expand ER refolding capacity via transcriptional programs that upregulate chaperones. The transcription factors X-box binding protein 1 (XBP-1), ATF6 p50, and ATF4 bind to nuclear ER stress-responsive elements (ERSEs) to upregulate chaperones including glucose-regulated protein 78/binding immunoglobulin protein (GRP78/BiP). Translation attenuation is promoted via phosphorylation of elongation initiation factor $2-\alpha$ (eIF $2 \alpha)$. The relative contributions of each UPR pathway to the proteostatic response are protein substrate- and cell-specific.

Ubiquitin-proteasome system. Misfolded or unassembled protein targets unresponsive to the UPR can be extracted from the ER by retrotranslocation, modified by covalent attachment of one or more lysine-48-linked (K-48-linked) polyubiquitin chains in the cytosol, and destroyed by the $26 \mathrm{~S}$ proteasome in a process known as ER-associated protein degradation (ERAD). The proteasome itself is composed of a cylinder-shaped catalytic core particle, the $20 \mathrm{~S}$ proteasome, which can be capped at either end by several different $19 \mathrm{~S}$ regulator complexes (reviewed in ref. 23). The $26 \mathrm{~S}$ proteasome is subject to failure as both the endogenous misfolded protein conformers and environmental factors, such as cigarette smoke, can directly impair proteasome activity (without affecting expression), resulting in cytosolic accumulation of mutant and bystander proteins. 




Proteostasis

Autophagy/mitophagy

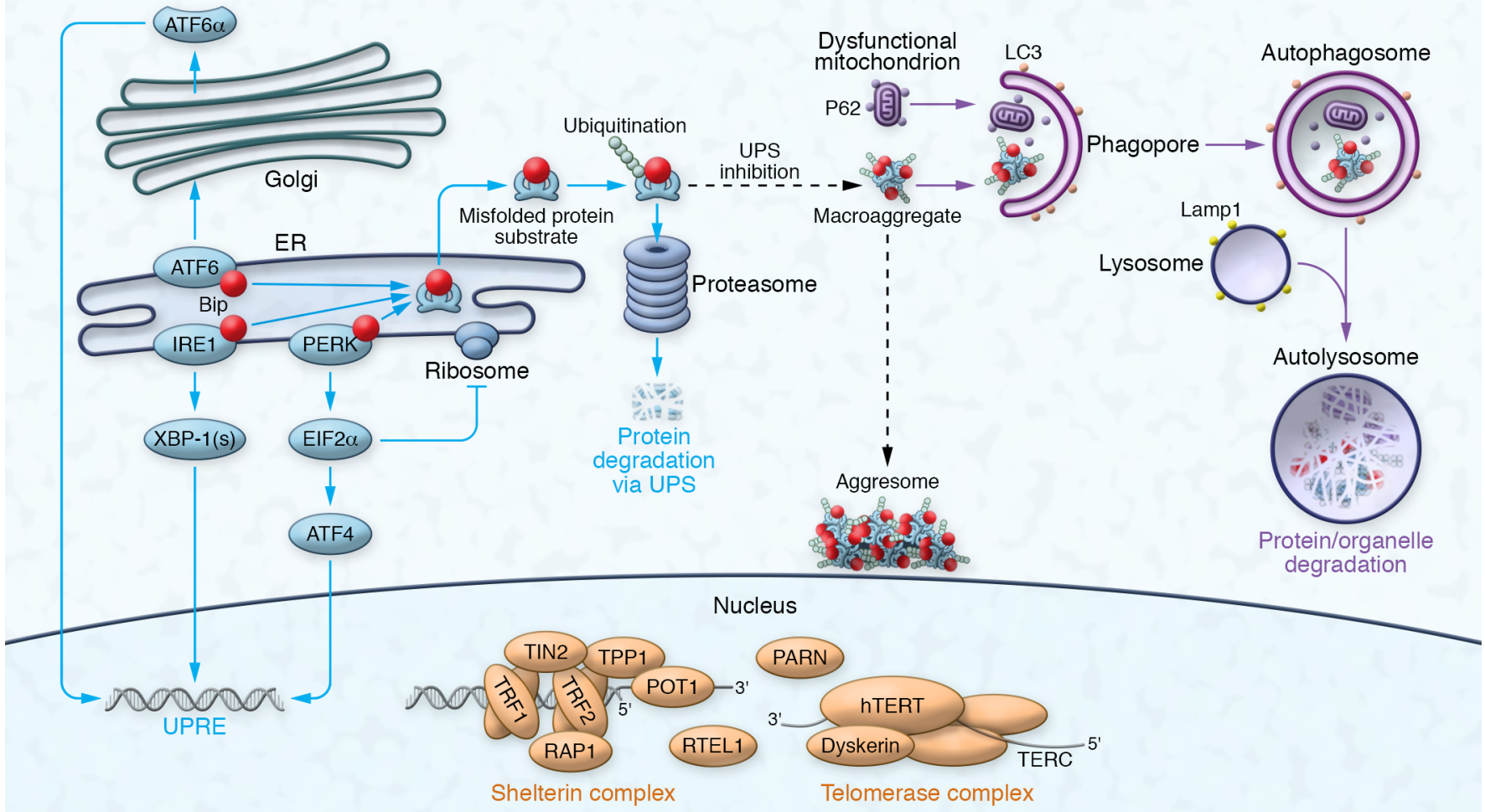

Telomere maintenance

Figure 2. AT2 cell quality control pathways: homeostasis for proteins, organelles, and DNA. Proteostasis (blue): Competitive binding of misfolded conformers to the molecular chaperone BiP activates one or more ER UPR sensors (ATF6, IRE1, PERK), initiating signaling to upregulate chaperones via three pathways: (i) ATF6p90 cleavage to ATF6p50 in the Golgi; (ii) IRE1 endoribonuclease activity for splicing XBP1; and (iii) PERK phosphorylation of elF2 $\alpha$, repressing translation and upregulating ATF4. Proteins refractory to refolding are retrotranslocated from the ER and targeted to the 265 proteasome for degradation by the UPS, via the ERAD process. UPS inhibition can promote the accumulation of cytosolic protein macroaggregates in the aggresome via a microtubule-dependent manner. Autophagy and mitophagy (purple): The autophagosome-lysosome system targets cytosolic protein aggregates (macroautophagy) and dysfunctional organelles, such as mitochondria (mitophagy), for degradation. Ubiquitin-binding receptors, such as p62/SQSTM1, recognize K-48-linked polyubiquitinated protein aggregates or K-63-linked polyubiquitin-tagged mitochondrial outer membrane proteins (initiated by PINK1 recruitment of the E3 ligase parkin). LC3 binding envelopes ubiquitinated cargo and leads to elongation of isolation membranes (phagophores) and maturation into autophagosomes. Fusion with LAMP1+ lysosomes results in an acidified and functional autophagolysosome (autolysosome) that degrades the internalized content. Telomere maintenance (orange): Telomere length relies on the interaction between the multiprotein shelterin complex (end protection), the telomerase holoenzyme (elongation), and the DNA helicase RTEL1. Shelterin is composed of telomeric repeat binding factors 1 and 2 (TRF1 and -2), Tin2, TPP1, Rap1, and POT1. Key telomerase components include a reverse transcriptase subunit (TERT), an RNA template (TERC), and dyskerin. PARN promotes TERC RNA maturation. Not depicted is the CST complex. This figure was adapted with permission from Mulugeta et al. (46).

Autophagy. While macroautophagy (hereafter, autophagy) was originally described as a cellular response to starvation, it is now recognized that, in a cell-autonomous fashion, autophagy functions as a second degradation pathway to remove aggregation-prone proteins (e.g., huntingtin, $\alpha$-synuclein) (24). Extensively discussed elsewhere $(25,26)$, in brief, autophagy is a dynamic process involving at least 30 known autophagy-relat- ed (Atg) proteins whose assembly and turnover ultimately result in the enclosure of cytosolic substrates and organelles within double-membrane vesicles (autophagosomes). Subsequently, fusion of the autophagosome with the lysosome triggers degradation of captured cargo. The highly regulated process receives inputs from a variety of sensing and signaling mechanisms, including mTOR1, class III PI3K, and several mTOR-indepen- 


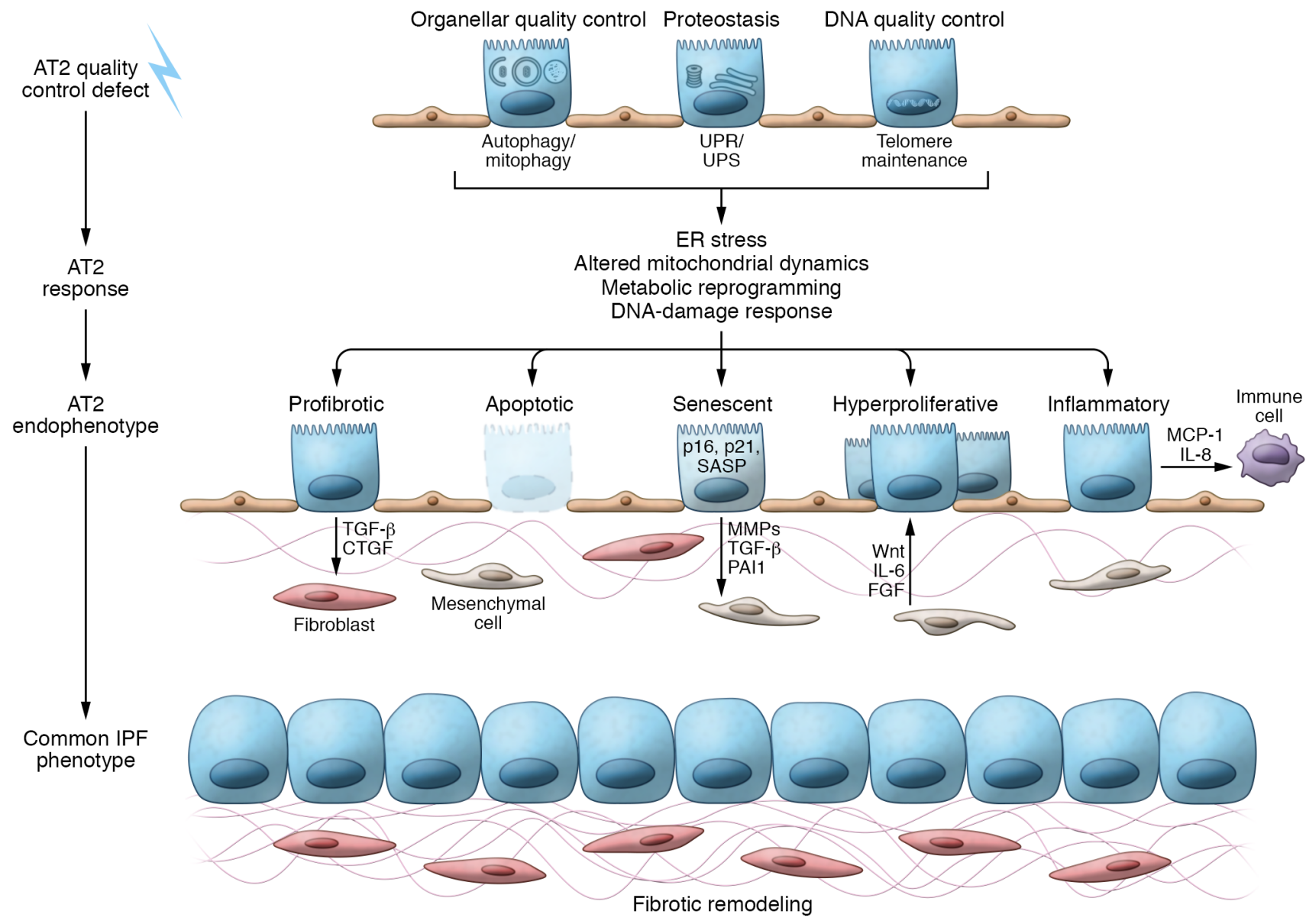

Figure 3. Cell quality control dysfunction and resulting endophenotypes that contribute to fibrotic remodeling. AT2 cell quality control defects: Effective AT2 cell quality control relies on management of malformed or misfolded proteins (proteostasis), degradation of dysfunctional organelles, and maintenance of telomere length. AT2 response: The loss of quality control involves AT2 cell adaptive compensations, such as activation of the UPR/UPS to regain proteostasis. However, sustained quality control defects lead to cellular disruption and injury from ER stress, persistent mitochondrial dysfunction, metabolic reprograming, and DNA damage responses. AT2 endophenotypes: Modeling AT2 cell quality control defects and interrogation of the IPF epithelia have identified a number of AT2 cell endophenotypes in the fibrotic lung. These include the production of profibrotic mediators including TGF- $\beta$, loss of AT2 cells through apoptosis, challenges to progenitor cell function resulting in the loss of progenitor capacity from senescence or emergence of hyperproliferative AT2 cells, and recruitment of immune cell populations. Common IPF phenotype: The resultant loss of alveolar architecture in IPF is defined by fibrotic remodeling and myofibroblast expansion into fibroblastic foci and hyperplasia of lung epithelial cells.

dent pathways. The selectivity of autophagy depends on the recognition of ubiquitinated aggregates by receptors, such as p62/ sequestosome-1 (p62/SQSTM1).

The AT2 cell places a heavy burden on both the UPR and the ubiquitin-proteasome system (UPS) to control mutant or misfolded protein conformers, and maintenance of these quality control systems is necessary for lung homeostasis. The loss of regulated protein folding by conditional deletion of the ER membrane complex Emc3 in the epithelia during lung development resulted in disruption of surfactant lipids, protein synthesis and packaging, and AT2 cell UPR activation, causing neonatal respiratory failure (27). Selective disruption of the UPS in AT2 cells by genetic deletion of RPT3 (Psmc4), an ATPase subunit of the 19S regulatory particle of the $26 \mathrm{~S}$ proteasome in mice, augmented AT2 death and lung injury resembling adult respiratory distress syndrome (28). In contrast, autophagy appears to selectively remove polyubiquitinated protein aggregates (aggrephagy) (29) and cellular organelles, such as dysfunctional mitochondria (known as mitophagy; discussed below).
Crosstalk between these systems provides functional compensation when either one is challenged (28). However, overall failure of this network can persistently activate the UPR, transitioning the affected cell to a state of ER stress, under which UPR signaling becomes maladaptive and promotes cell dysfunction and death (30).

\section{Organellar quality control: mitophagy}

In addition to aggrephagy, AT2 cells use autophagy to selectively target and remove individual subcellular components, including invading pathogens (xenophagy), lipids (lipophagy), and dysfunctional cellular organelles, such as ER or lysosome-related organelles. Best known among these is the degradation of dysfunctional mitochondria, termed "mitophagy," which is part of a larger cellular system that orchestrates the turnover of mitochondrial mass. Remarkably specific, mitophagy requires the coordination of cytosolic factors and signals assembled at the outer mitochondrial membrane to identify and target dysfunctional mitochondria to the autophagosome (reviewed in refs. 31, 32). 
Key steps in the process include stabilization of PTEN-induced putative kinase 1 (PINK1) in the outer mitochondrial membrane in response to lowered transmembrane potential; recruitment of the E3 ligase parkin, resulting in K-63-linked polyubiquitination of a variety of mitochondrial protein substrates (e.g., mitofusins [MFN1, MFN2]); and sequestration of ubiquitin-decorated mitochondria in the same autophagic machinery commissioned for protein removal. Knockout of Pink1 in AT2 cells in mice results in a dysmorphic mitochondrial phenotype and an age-dependent susceptibility to fibrotic lung remodeling after challenge with viral infection (33). Perturbations in PINK1 expression and mitophagy (31) appear in the IPF AT2 cell (discussed below).

\section{DNA quality control: telomere homeostasis}

Telomeres are DNA/protein caps at the ends of chromosomes that are essential to genome stability (reviewed in refs. 34, 35). Because of what is known as the "end replication problem," telomeres shorten with each cell division, and when they become too short, a DNA damage checkpoint response triggers a permanent cell cycle arrest or apoptosis (36). Telomeres are maintained by telomerase, composed of a reverse transcriptase (telomerase reverse transcriptase [TERT]) that synthesizes new telomeres from an endogenously encoded RNA template (telomerase RNA [TR]). Telomerase expression is tightly controlled such that only a few cell types have telomerase activity. Within the adult mouse lung AT2 cell pool, a telomerase-positive subpopulation after lung injury has been identified, perhaps signifying a progenitor subpopulation (37); and induced AT2 cell telomere dysfunction caused alveolar progenitor senescence (38). Foreshortened telomeres are causally associated with a spectrum of age-related diseases including bone marrow failure, liver fibrosis, and lung fibrosis (39, 40). Spatial interrogation of the fibrotic lung has revealed considerably shorter telomere length in AT2 cells in regions of fibrosis (41).

\section{Profibrotic AT2 endophenotypes and disrupted cell quality control}

Sustained disruption of one or more AT2 quality control repertoires results in the development of profibrotic epithelial endophenotypes, depicted in Figure 3.

\section{Disrupted proteostasis: surfactant mutations as proof of concept}

Interrogation of AT2 cells from IPF explants using immunohistochemistry $(17,42)$ and transcriptional profiling $(43)$ has identified molecular signals of challenged proteostasis including UPR activation, ER stress, and impaired autophagy. Deconvoluting the precise role of specific epithelial UPR pathways in lung fibrosis has been challenging. Recently, AT2-specific deletion of a key regulator of ER homeostasis, the chaperone GRP78/BiP, resulted in impaired AT2 progenitor capacity, activated TGF- $\beta$ signaling, and spontaneous age-dependent fibrotic lung remodeling with features of UIP pathology (44).

Perhaps no better proof of concept for the role of AT2 quality control dysfunction as a driver of lung fibrosis exists than lessons learned from mutations in surfactant proteins (SPs). The alveolar epithelial surface is coated by surfactant, a complex mixture of lipids, and SPs that in combination reduce surface tension at the alveolar air-liquid interface (45). Of the four major SPs, the hydrophobic
SP-B and SP-C, which participate in surface tension modulation, are secreted exclusively by AT2 cells. Over 60 mutations in the AT2 cell-restricted SFTPC gene have been identified in pediatric patients with childhood interstitial lung disease (ChILD) and adults with familial interstitial pneumonias (FIPs) or IPF (46-48). While SFTPC mutations appear in less than $5 \%$ of sporadic IPF patients, these highly penetrant, rare mutants with high effect sizes have enhanced our understanding of AT2 cell biology in lung fibrosis.

Mutant SFTPC expression induces two predominant AT2 quality control phenotypes:

ERAD-UPS challenged with ER stress. The first two diseaseassociated SFTPC mutations identified, and the majority described subsequently, localize to the proSP-C COOH-terminal (BRICHOS) domain and disrupt a key intra-propeptide disulfide bond within the domain (49). When expressed in vitro, SFTP$C^{B R I C H O S}$ mutants are ER retained, inhibit the proteasome, form cytosolic aggregates, and activate the UPR. Similar markers of UPR/ER stress can be found in AT2 cells of patients carrying such mutations. Murine models of SFTPC ${ }^{\text {BRICHOS }}$ mutants have collectively defined a toxic gain of function linking a dysfunctional AT2 cell phenotype and fibrosis (50-52). Conditional expression of the clinical SFTPC ${ }^{L 188 Q}$ mutation produced AT2 ER stress and enhanced sensitivity to bleomycin-induced fibrosis (51). More recently, induced expression of the clinical SP-C mutant $\left(S f t p c^{C 121 G}\right.$ ) knocked into the mouse $S f t p c$ locus led to notable AT2 ER stress and apoptosis with spontaneous fibrotic remodeling (52). In total, these models provide fundamental support for AT2 ER stress and the resultant cytokine elaboration, apoptosis, and disrupted progenitor cell function as upstream drivers of lung fibrosis.

Autophagy impaired. The most common SFTPC mutation identified in the clinic results in a threonine-to-isoleucine substitution at the 73rd codon of the SFTPC allele $\left(\right.$ SFTPC $\left.{ }^{173 T}\right)$, corresponding to a region of the SP-C proprotein termed the linker domain. In contrast to BRICHOS isoforms, in vitro expression of linker mutations results in their mistrafficking and accumulation in the late endosomal pathway, resulting in autophagy blockade and impaired mitophagy (53). Preclinical modeling using an Sftpc $c^{173 T}$ allelic knockin phenocopied both the cellular and the clinical phenotypes, including a mistrafficked proSP-C isoform, impaired AT2 autophagy, spontaneous alveolitis, and UIP-like fibrotic remodeling, further validating the role of dysfunctional AT2 quality control as an upstream component of IPF (54-56).

Genetic analyses of FIP kindreds have also identified mutations in surfactant protein A genes (SFTPA1, SFTPA2) (57-59). In vitro, expression of disease-associated SFTPA2 mutations produced proximally retained SP-A isoforms and UPR activation (60). Recent in vivo expression of a disease-associated SFTPA1 mutation resulted in AT2 UPR activation, necroptosis, and spontaneous lung fibrosis linked by ER stress-induced JNK signaling $(61,62)$.

Beyond genetic susceptibility, IPF is a disease of both aging (63) and environment. As proteostatic capacity declines with age in a variety of organs (notably the brain), the assaults of aging and environmental exposures in IPF likely converge at the level of AT2 quality control. Supportive of this paradigm, older mice show increased susceptibility to herpesvirus-induced lung fibrosis with corresponding increases in ER stress markers and apoptosis (64). Environmental exposures associated with human IPF, including cigarette smoke, 
inhaled particulates, and asbestos exposure, all of which impair proteasome activity, increase epithelial ER stress, and/or raise the susceptibility to bleomycin-induced fibrosis in mice (65-67).

\section{Disrupted organelle homeostasis}

Cytoplasmic multilamellated organelles, lamellar bodies (LBs), are the storage vesicles from which surfactant is released into the alveolus and are a defining feature of the mature AT2 cell (12). LBs are part of the lysosome-related organelle (LRO) family and are intimately involved in surfactant homeostasis, interfacing with surfactant exocytosis, endocytosis, and recycling (68).

Disruption of LB homeostasis promotes AT2 cell dysfunction and fibrotic lung remodeling and is best exemplified by Hermansky-Pudlak syndrome (HPS), a rare autosomal recessive disorder caused by mutations in cell adapter protein (AP) complexes such as AP3 or in biogenesis of lysosome-related organelles complexes (BLOC-1, -2, -3). HPS patients demonstrate marked impairments in biogenesis and function of LROs including platelet granules and melanosomes, with three HPS subtypes (1,2, and 4) also marked by the presence of giant LBs and early pulmonary fibrosis with UIP features (69). Recent in vitro modeling has helped define the link between loss of organelle homeostasis and HPS AT2 cell-driven lung fibrosis. Gene-edited stable HPS mouse cell lines (70), embryonic stem cells expressing HPS genes (71), and patient-specific induced pluripotent stem cell-derived AT2 cells (72) each exhibit disruption of LB homeostasis and provide proof of concept of their role as hubs for epithelial-immune cell and epithelial-mesenchymal crosstalk. These HPS AT2 models elaborate proinflammatory cytokines (e.g., MCP-1), complementing HPS mouse models that demonstrate AT2-mediated recruitment of profibrotic monocyte/macrophage lineages (73). In addition, AT2 cells were recently shown to serve as a source of both the profibrotic cytokine IL-11 $(71,74,75)$ and matrix metalloproteinases (76).

Clinical mutations in $A B C A 3$, an ATP-dependent transporter of phosphatidylcholine and cholesterol into LROs that is critical for LB formation, further connect LB dysfunction to fibrotic lung disease. Homozygous null $A B C A 3$ mutations result in absence of LBs and in neonatal respiratory failure in both humans and mice (77-82). Beyond loss of function, a number of clinical $A B C A 3$ mutations have been associated with pulmonary fibrosis (58). As with SFTPC, cellular phenotypes from $A B C A 3$ mutations are based on protein behavior, with hypomorphic, ER-retained, and mistrafficked isoforms found (46). However, mechanistic links between $A B C A 3$ mutants, cell quality control alterations, and fibrosis await further study (83).

\section{Altered AT2 metabolomics and mitochondrial dynamics}

While much focus on bioenergetics and metabolism in IPF has centered on the fibroblast, recent transcriptomic analysis of IPF epithelia has identified distinct metabolic pathway changes in AT2 cells $(43,84)$. The functional importance of a potential metabolic shift remains undefined; however, pathways driving these changes could include alterations in mitochondrial biomass or function. The regulation of mitochondrial biogenesis, assembly (fusion), disassembly (fission), and clearance (mitophagy), collectively termed mitochondrial dynamics, adapts ATP production to situational needs such as enhanced biosynthetic requirements or progenitor cell function (reviewed in ref. 85). Alterations in mitochondrial biogenesis and mitophagy are features of aging (86), and these same molecular footprints are found in AT2 cells of IPF patients (33). Interestingly, in vitro and in vivo models that express the aforementioned SFTPC mutation, $S f t p c^{I 73 T}$, display commensurate loss of mitochondrial transmembrane potential $(\Delta \psi)$, increased mitochondrial biomass, and impaired mitophagy $(53,54)$. Chemically induced AT2 ER stress produces a decrease in PINK1, thus linking a more global dysfunction in the AT2 quality control of malformed proteins with impaired mitochondrial homeostasis in IPF (87). Additional consequences of disrupted mitochondrial dynamics beyond energy production include AT2 cell oxidative injury and mitochondrial DNA (mtDNA) release $(85,88)$. Circulating mtDNA has recently emerged as a promising biomarker in IPF (89).

The bioenergetic consequences of epithelial dysfunction, be it from ER stress or impaired mitochondria homeostasis, are largely unexplored. The first bioenergetic functional assessment in the distal lung epithelia used both bleomycin and TGF- $\beta$ mouse models to demonstrate decreases in AT2 mitochondrial membrane potential and oxygen consumption (90). Mechanistically these changes were traced to alterations in AT2 thyroid hormone metabolism, as iodothyronine deiodinase (DIO2), which converts prohormone thyroxine (T4) to the active 3,5,3'-triiodothyronine (T3) form, was increased in the IPF lung. As proof of concept, thyroid hormone mimetics, acting through PPAR $\gamma$ coactivator $1 \alpha$ (PGC$1 \alpha$ ), a master regulator of mitochondrial biogenesis, inhibited fibrosis. While the observed AT2 mitochondrial and bioenergetic changes await translation to human IPF, hypothyroidism has been associated with an unfavorable prognosis in IPF (91).

\section{Telomere attrition and senescence}

Telomere shortening activates a DNA damage response pathway that triggers cellular senescence, a state of permanent cell cycle arrest, and has been implicated in the pathogenesis of many chronic diseases of aging and dysfunctional tissue repair, including IPF (92). In multiple studies, interrogation of telomere length as a risk factor has shown that patients with either IPF or other fibrotic diffuse parenchymal lung diseases (DPLDs) are more likely to have shortened telomeres than age-matched controls. Shortened telomere length also portends worse outcomes in IPF $(40,93,94)$. While peripheral blood mononuclear cell telomere length has become the clinical surrogate for telomere length (95), short telomeres in AT2 cells were found both in patients with telomere gene mutations and in sporadic IPF with telomere shortening (40).

While mutations in telomerase components and maintenance genes appear in only a small portion of sporadic IPF patients, mutations in $h T E R T, R T E L 1$, and $h T R$ commonly occur in patients with a family history of $\operatorname{DPLD}(40,96,97)$. In vivo modeling of clinical mutations has produced variable results. Global deletion of Tert and the induction of critically shortened telomeres in the epithelium did not result in spontaneous lung fibrosis and yielded variable responses to bleomycin injury (98-100). Conversely, induction of a substantial telomere-DNA damage response due to conditional AT2 deletion of shelterin complex genes $(\operatorname{Tr} f 2, \operatorname{Tr} f 1)$ does support a role for AT2 telomere 
quality control in lung fibrosis: Two groups have demonstrated that deletion of $\operatorname{Trf} 1$ in the AT2 cells results in age-dependent spontaneous lung fibrosis $(100,101)$, and deletion of $\operatorname{Tr} f 2$ resulted in AT2 senescence with impaired proliferative capacity, spontaneous lung inflammation, and fatal injury following bleomycin, which were not attributed to increased AT2 apoptosis (38). While the aforementioned AT2 cell telomere shortening can contribute to senescence, perturbation of mitochondrial homeostasis or activation of ER stress pathways may also contribute to this deleterious AT2 endophenotype $(102,103)$.

Markers of cellular senescence (p21, p53) have been identified in epithelia around fibrotic foci in IPF biopsies (104). Single-cell transcriptional profiling of the IPF lung has identified enrichment of senescence-associated pathways within the distal lung epithelial pool $(84,105)$. The mechanisms by which AT2 senescence contributes to fibrotic remodeling remain ill-defined but are likely related to both the transition to a senescence-associated secretory phenotype (SASP) and loss of epithelial repair/progenitor capacity. SASP is associated with production of chemokines and cytokines that are implicated in crosstalk with immune cells as well as factors that are associated with mesenchymal crosstalk and tissue remodeling. In bleomycin-challenged mice, distal lung epithelial cells increase expression of profibrotic SASP factors, including MCP-1, matrix metalloproteinases, and TGF- $\beta$ (105). Recent modeling of AT2 senescence by conditional deletion of Sin3a, a key component of the Sin3-HDAC complex, resulted in p53-dependent cellular senescence, AT2 cell depletion, and TGF- $\beta$-dependent spontaneous pulmonary fibrosis, further linking AT2 SASP signaling to fibrosis (106).

\section{Contributions of other distal epithelial lineages to IPF}

\section{AT1 cells}

The majority of the alveolar surface is covered by AT1 cells, whose thin squamous morphology and intimate contact with the adjacent capillary plexus permit efficient gas exchange. While loss of AT1 cells is a cardinal feature of IPF histology (107), how these cells contribute to fibrosis, including the inciting event that promotes their disappearance and how AT2 progenitor dysfunction is involved in AT1 dynamics, remains unclear. Single-cell transcriptomics from the IPF lung has identified cells elaborating markers of both AT2 and AT1 cells, revealing a subset of epithelial cells positioned as transitional cells $(43,108)$. Recent reports using bleomycin modeling and organoid culture identified and defined a homologous aberrant transitional cell in the mouse lung during fibrosis defined by Krt8 expression and activation of p53 and senescence pathways $(109,110)$. Determining the factors that modulate complete AT2 transdifferentiation may provide additional insight into aberrant lung repair.

\section{Distal airway epithelial populations}

The conducting airways of the distal human lung are composed of a complex, diverse set of secretory, mucin-producing, and multiciliated epithelial cells that transition to a low cuboidal morphology at the respiratory bronchiole, ultimately intermixing with gas-exchanging alveoli (reviewed in refs. 111, 112). While recent focus has emphasized defects in the alveolar compartment as a primary driver, there are key pieces of evidence implicating potential contributions of proximal airway epithelial cells in IPF pathogenesis (113-116). To date, key populations identified include the following:

Mucin-producing cells. A defining histological feature of IPF is the honeycomb cyst, a mucin-containing cystic airway structure lined with airway epithelium in the periphery of the fibrotic lung (5). Linkage analysis has identified a minor-allele SNP (rs35705950 “T" allele) in the upstream promoter of MUC5B that confers a 4-fold increase in susceptibility to IPF and is associated with increases in both $M U C 5 B$ expression and mucin staining $(114,117)$. The MUC5B allele has also emerged as a risk factor for development of radiographic interstitial lung abnormalities (118). Recent identification that upregulation of the IRE1 pathway through XBP-1 in the airway epithelia differentially activates the MUC5B promoter carrying the rs35705950 T allele suggests a link between airway cell UPR activation and enhanced mucin production (119). However, mice overexpressing Muc5b in AT2 cells or conducting airways failed to support its role as a primary driver of spontaneous fibrosis (120). This model did, however, exhibit enhanced sensitivity to bleomycin.

Basal cells and transitional epithelial cells. Ectopic populations potentially contributing to honeycomb bronchiolarization include lineage-negative epithelial progenitor cells (LNEPs), also referred to as distal airway stem cells (DASCs) $(116,121)$. LNEPs express the canonical basal cell genes Krt5 and Trp63, and lineage tracing experiments in mice show that this rare population of distal airway cells migrate to the alveolar compartment and form pods resembling honeycomb cysts in areas of substantial injury (e.g., after influenza). LNEPs are responsive to Notch signaling driven by local lung hypoxia through the hypoxia-inducible factor HIF$1 \alpha$. The identification of a distal lung epithelial subpopulation with activated Notch signaling in fibrotic human lungs suggests participation of dysplastic LNEPs in an aberrant repair program, perhaps activated to reconstitute a void in the distal lung left by AT2 progenitor failure (122).

Single-cell RNA sequencing of the IPF lung has also identified an aberrant transitional epithelial cell state with a putative origin in the distal airways defined by expression of markers of both basal cells (KRT17) and distal epithelial lineages (e.g., SOX9, NAPSA) and absence of SOX2 $(108,123)$. Trajectory analysis suggests that the precursor for this transitional epithelial cell is a population intermediate between the $S C G B 3 A 2^{+}$epithelia in respiratory bronchioles and a transitional AT2 cell state. While its role in IPF has not been established, it localizes to the epithelial layer covering fibroblastic foci and coexpresses markers of epithelial-mesenchymal transition (COL1A1, FN1, TNC), the biomarker MMP7, and $\alpha_{v} \beta_{6}$ integrin subunits (ITBAV, ITGB6), suggesting a functional role in niche homeostasis and TGF- $\beta$ activation.

\section{Epithelial participation in IPF crosstalk networks}

The route from epithelial dysfunction to lung fibrosis requires contributions from multiple cell types (Figure 1). Identifying and interrogating the crosstalk between the epithelium and both immune cells and the mesenchyme is paramount to effectively identify targets improve IPF therapeutics. 


\section{AT2-immune cell crosstalk}

The role of the AT2 cell in recruiting immune effector cells, including diverse macrophage populations and, in particular, the CCR2 ${ }^{+}$ monocyte population, is now recognized as a key proximal event in lung fibrogenesis. The ligand for CCR2, MCP-1, was identified in the epithelium of IPF explants a decade before the recognition that a bone marrow-derived monocyte population played a critical role in the acceleration of fibrosis in bleomycin-treated mice (124-126). The specific contribution of the AT2 cell to the CCL2/ CCR2 epithelial-macrophage axis required the development of preclinical models such as Sftpc and HPS mice (52, 54, 73, 127, 128). In both models, disruption of the MCP-1/CCR2 axis attenuated fibrosis. To date, successful targeting of this axis in IPF has been challenging, perhaps owing to the redundancy in ligands and receptors responsible for monocyte recruitment $(52,129)$.

An additional critical reason for understanding epithelial-immune cell axes lies in the realization that, in some patients, the normal course of progressive fibrosis and lung function decline may become punctuated by periods of substantial inflammation often preceded by epithelial injury, be it by aspiration, viral infection, or environmental exposure (130-133). Clinically defined by appearance of new ground-glass opacities on chest radiography, a polycellular alveolitis, and histologically apparent diffuse alveolar damage (134), these events, termed "acute exacerbations" (AEs) of IPF, portend a poor prognosis, with a roughly $50 \%$ in-hospital mortality and a rapid acceleration in the underlying fibrosis in survivors (135). Sera of patients experiencing IPF AEs demonstrate markers of epithelial injury and activation (136), as well as a number of putative lung epithelia-derived cytokines associated with recruitment of both monocyte and granulocyte populations $(137,138)$.

\section{Epithelial-mesenchymal signaling}

Multiple modes of epithelial-mesenchymal crosstalk exist in the fibrotic niche, including epithelial cell-derived growth factors, integrins, TGF- $\beta$ signaling, and alterations in extracellular matrix causing release of matrikines. These signaling pathways are thought to accelerate the recruitment and activation of fibroblasts toward myofibroblast differentiation.

TGF- $\beta$ signaling, pivotal in tempering tissue inflammation $(139,140)$, has been identified in the epithelia of patients with IPF (141). The importance of TGF- $\beta$ for fibrogenesis has been affirmed using transgenic mouse models, where its overexpression in the distal lung epithelia produces aberrant remodeling marked by an amassing of myofibroblasts (20). The recent identification of Tgfb enrichment in AT2 cells in fibrosis models induced either by expression of $S f t p c$ mutations or by genetic induction of AT2 senescence highlights a role for early AT2 cell production of TGF- $\beta(52,54$, 106). Additionally, a recent study identifying mechanical stretch as an upstream regulator of both autocrine and paracrine TGF- $\beta$ sig- naling suggests that the AT2 cell may be a source of alveolar niche TGF- $\beta$ even in the absence of quality control malfunction (142).

Increasingly, AT2 epithelial-mesenchymal signaling is thought to be bidirectional with fibroblast-driven epithelial proliferation. The recent identification of mesenchymal heterogeneity in the airway and alveolar compartment by multiple groups refines our understanding of this crosstalk during injury and repair (143, 144). Although contextualization of recently identified FGF, WNT, BMP, and IL-6/STAT3 signaling pathways between mesenchymal subpopulations and the distal lung epithelium in the setting of fibrotic remodeling has not been rigorously studied, consideration of these dynamics may provide additional upstream targets for IPF treatment.

\section{Conclusions}

While dysfunction in cellular quality control pathways has been well recognized as an important driver of neurodegenerative diseases, cancer, and aging, disruptions of this same network in the distal lung epithelium are only recently emerging as important pathogenic mechanisms and therapeutic targets for pulmonary fibrosis. Improved understanding of how cell quality control defects result in the emergence of aberrant AT2 cell endophenotypes and profibrotic signaling in the alveolar niche will permit new insights into the proximal events that lead to clinical IPF, as well as open up many new opportunities for novel drug discovery.

\section{Author contributions}

MFB and JK developed the concept; JK drafted the manuscript; MFB edited the manuscript. Both authors reviewed and approved the final version before submission.

\section{Acknowledgments}

We thank members of the Beers laboratory for their critical comments and numerous collaborators who have shaped our thinking. We apologize to the many colleagues whose work could not be cited in detail due to space constraints. This work was supported by VA Merit Review 1I01BX001176 (to MFB), NIH R01 HL145408 (to MFB), NIH 2 P30 ES013508 (to MFB), and the Francis Family Foundation Parker B. Francis Fellowship (to JK). JK was supported in part by NIH K08 HL150226 and is a PFF Scholar of the Pulmonary Fibrosis Foundation. MFB is an Albert M. Rose Established Investigator of the Pulmonary Fibrosis Foundation. The content of this article does not represent the views of the US Department of Veterans Affairs or the United States Government.

Address correspondence to: Michael F. Beers, Pulmonary, Allergy, and Critical Care Division, University of Pennsylvania School of Medicine, Suite 216 Edward J. Stemmler Hall, 3450 Hamilton Walk, Philadelphia, Pennsylvania 19104-5159, USA. Phone: 215.898.9106; Email: mfbeers@pennmedicine.upenn.edu.

\footnotetext{
1. Travis WD, et al. An official American Thoracic Society/European Respiratory Society statement: Update of the international multidisciplinary classification of the idiopathic interstitial pneumonias. Am J Respir Crit Care Med. 2013;188(6):733-748.
}
2. Chambers DC, et al. The International Thoracic Organ Transplant Registry of the Internationa Society for Heart and Lung Transplantation: Thirty-sixth adult lung and heart-lung transplan- tation Report-2019; Focus theme: Donor and recipient size match. J Heart Lung Transplant.

2019;38(10):1042-1055.

3. Raghu G, Chen SY, Hou Q, Yeh WS, Collard HR. Incidence and prevalence of idiopathic pulmonary fibrosis in US adults 18-64 years old. Eur Respir J. 2016;48(1):179-186.

4. Lederer DJ, Martinez FJ. Idiopathic pulmonary 
fibrosis. N Engl JMed. 2018;378(19):1811-1823.

5. Seibold MA, et al. The idiopathic pulmonary fibrosis honeycomb cyst contains a mucocilary pseudostratified epithelium. PLoS One. 2013;8(3):e58658.

6. Flaherty KR, et al. Histopathologic variability in usual and nonspecific interstitial pneumonias. $A m$ J Respir Crit Care Med. 2001;164(9):1722-1727.

7. Nathan SD, et al. Long-term course and prognosis of idiopathic pulmonary fibrosis in the new millennium. Chest. 2011;140(1):221-229.

8. Dempsey TM, Sangaralingham LR, Yao X, Sanghavi D, Shah ND, Limper AH. Clinical effectiveness of antifibrotic medications for idiopathic pulmonary fibrosis. Am J Respir Crit Care Med. 2019;200(2):168-174.

9. King TE, et al. A phase 3 trial of pirfenidone in patients with idiopathic pulmonary fibrosis. $N$ EnglJMed. 2014;370(22):2083-2092.

10. Richeldi L, et al. Efficacy and safety of nintedanib in idiopathic pulmonary fibrosis. $N$ Engl J Med. 2014;370(22):2071-2082.

11. Haschek WM, Witschi H. Pulmonary fibrosis-a possible mechanism. Toxicol Appl Pharmacol. 1979;51(3):475-487.

12. Beers MF, Moodley Y. When is an alveolar type 2 cell an alveolar type 2 cell? A conundrum for lung stem cell biology and regenerative medicine. $\mathrm{Am}$ J Respir Cell Mol Biol. 2017;57(1):18-27.

13. Barkauskas CE, et al. Type 2 alveolar cells are stem cells in adult lung. J Clin Invest. 2013;123(7):3025-3036.

14. Sisson TH, et al. Targeted injury of type II alveolar epithelial cells induces pulmonary fibrosis. Am J Respir Crit Care Med. 2010;181(3):254-263.

15. Garcia O, et al. Targeted type 2 alveolar cell depletion. A dynamic functional model for lung injury repair. Am J Respir Cell Mol Biol. 2016;54(3):319-330.

16. Thannickal VJ, Horowitz JC. Evolving concepts of apoptosis in idiopathic pulmonary fibrosis. Proc Am Thorac Soc. 2006;3(4):350-356.

17. Korfei M, et al. Epithelial endoplasmic reticulum stress and apoptosis in sporadic idiopathic pulmonary fibrosis. Am J Respir Crit Care Med. 2008;178(8):838-846.

18. Uhal BD, Joshi I, Hughes WF, Ramos C, Pardo A, Selman M. Alveolar epithelial cell death adjacent to underlying myofibroblasts in advanced fibrotic human lung. Am J Physiol. 1998;275(6):L1192-L1199.

19. Wallach-Dayan SB, Izbicki G, Cohen PY, Gerstl-Golan R, Fine A, Breuer R. Bleomycin initiates apoptosis of lung epithelial cells by ROS but not by Fas/FasL pathway. Am J Physiol Lung Cell Mol Physiol. 2006;290(4):L790-L796.

20. Lee CG, et al. Early growth response gene 1-mediated apoptosis is essential for transforming growth factor beta1-induced pulmonary fibrosis. JExp Med. 2004;200(3):377-389.

21. Balch WE, et al. Malfolded protein structure and proteostasis in lung diseases. Am J Respir Crit Care Med. 2014;189(1):96-103.

22. Balch WE, Morimoto RI, Dillin A, Kelly JW. Adapting proteostasis for disease intervention. Science. 2008;319(5865):916-919.

23. Meiners S, Keller IE, Semren N, Caniard A. Regulation of the proteasome: evaluating the lung proteasome as a new therapeutic target. Antioxid Redox Signal. 2014;21(17):2364-2382.

24. Klionsky DJ, Emr SD. Autophagy as a regulated pathway of cellular degradation. Science. 2000;290(5497):1717-1721.

25. Levine B, Kroemer G. Autophagy in the pathogenesis of disease. Cell. 2008;132(1):27-42.

26. Choi AM, Ryter SW, Levine B. Autophagy in human health and disease. N EnglJMed. 2013;368(7):651-662.

27. Tang X, et al. EMC 3 coordinates surfactant protein and lipid homeostasis required for respiration. JClin Invest. 2017;127(12):4314-4325.

28. Sitaraman S, Na CL, Yang L, Filuta A, Bridges JP, Weaver TE. Proteasome dysfunction in alveolar type 2 epithelial cells is associated with acute respiratory distress syndrome. Sci Rep. 2019;9(1):12509.

29. Zhao J, Goldberg AL. Coordinate regulation of autophagy and the ubiquitin proteasome system by MTOR. Autophagy. 2016;12(10):1967-1970.

30. Kropski JA, Blackwell TS. Endoplasmic reticulum stress in the pathogenesis of fibrotic disease. JClin Invest. 2018;128(1):64-73.

31. Youle RJ, Narendra DP. Mechanisms of mitophagy. Nat Rev Mol Cell Biol. 2011;12(1):9-14.

32. Youle RJ, van der Bliek AM. Mitochondrial fission, fusion, and stress. Science. 2012;337(6098):1062-1065.

33. Bueno M, et al. PINK1 deficiency impairs mitochondrial homeostasis and promotes lung fibrosis. JClin Invest. 2015;125(2):521-538.

34. Cech TR. Beginning to understand the end of the chromosome. Cell. 2004;116(2):273-279.

35. Blackburn EH, Greider CW, Szostak JW. Telomeres and telomerase: the path from maize, Tetrahymena and yeast to human cancer and aging. Nat Med. 2006;12(10):1133-1138.

36. d'Adda di Fagagna F, et al. A DNA damage checkpoint response in telomere-initiated senescence. Nature. 2003;426(6963):194-198.

37. Driscoll B, Buckley S, Bui KC, Anderson KD, Warburton D. Telomerase in alveolar epithelial development and repair. Am J Physiol Lung Cell Mol Physiol. 2000;279(6):L1191-L1198.

38. Alder JK, et al. Telomere dysfunction causes alveolar stem cell failure. Proc Natl Acad Sci U S A. 2015;112(16):5099-5104.

39. Armanios M. Telomeres and age-related disease: how telomere biology informs clinical paradigms. J Clin Invest. 2013;123(3):996-1002.

40. Alder JK, et al. Short telomeres are a risk factor for idiopathic pulmonary fibrosis. Proc Natl Acad Sci U S A. 2008;105(35):13051-13056.

41. Snetselaar R, et al. Short telomere length in IPF lung associates with fibrotic lesions and predicts survival. PLoS One. 2017;12(12):e0189467.

42. Lawson WE, et al. Endoplasmic reticulum stress in alveolar epithelial cells is prominent in IPF: association with altered surfactant protein processing and herpesvirus infection. Am JPhysiol Lung Cell Mol Physiol. 2008;294(6):L1119-L1126.

43. Xu Y, et al. Single-cell RNA sequencing identifies diverse roles of epithelial cells in idiopathic pulmonary fibrosis. JCI Insight. 2016;1(20):e90558.

44. Borok Z, et al. Grp78 loss in epithelial progenitors reveals an age-linked role for endoplasmic reticulum stress in pulmonary fibrosis. Am J Respir Crit
Care Med. 2020;201(2):198-211.

45. Pérez-Gil J. Structure of pulmonary surfactant membranes and films: the role of proteins and lipid-protein interactions. Biochim Biophys Acta. 2008;1778(7-8):1676-1695

46. Mulugeta S, Nureki S, Beers MF. Lost after translation: insights from pulmonary surfactant for understanding the role of alveolar epithelial dysfunction and cellular quality control in fibrotic lung disease. Am J Physiol Lung Cell Mol Physiol. 2015;309(6):L507-L525.

47. Nogee LM, Dunbar AE, Wert SE, Askin F, Hamvas A, Whitsett JA. A mutation in the surfactant protein $\mathrm{C}$ gene associated with familial interstitial lung disease. $N$ Engl J Med. 2001;344(8):573-579.

48. Thomas AQ, et al. Heterozygosity for a surfactant protein $\mathrm{C}$ gene mutation associated with usual interstitial pneumonitis and cellular nonspecific interstitial pneumonitis in one kindred. Am J Respir Crit Care Med. 2002;165(9):1322-1328.

49. Willander $\mathrm{H}$, et al. High-resolution structure of a BRICHOS domain and its implications for anti-amyloid chaperone activity on lung surfactant protein C. Proc Natl Acad Sci U S A. 2012;109(7):2325-2329.

50. Bridges JP, Wert SE, Nogee LM, Weaver TE. Expression of a human surfactant protein $\mathrm{C}$ mutation associated with interstitial lung disease disrupts lung development in transgenic mice. J Biol Chem. 2003;278(52):52739-52746.

51. Lawson WE, et al. Endoplasmic reticulum stress enhances fibrotic remodeling in the lungs. Proc Natl Acad Sci U S A. 2011;108(26):10562-10567.

52. Katzen J, et al. An SFTPC BRICHOS mutant links epithelial ER stress and spontaneous lung fibrosis. JCI Insight. 2019;4(6):126125.

53. Hawkins A, et al. A non-BRICHOS SFTPC mutant (SP-CI73T) linked to interstitial lung disease promotes a late block in macroautophagy disrupting cellular proteostasis and mitophagy. Am J Physiol Lung Cell Mol Physiol. 2015;308(1):L33-L47.

54. Nureki SI, et al. Expression of mutant Sftpc in murine alveolar epithelia drives spontaneous lung fibrosis. JClin Invest. 2018;128(9):4008-4024.

55. Blackwell TS. Lung injury and fibrosis induced by a mutant form of surfactant protein C. J Clin Invest. 2018;128(9):3745-3746.

56. Albert RK, Schwartz DA. Revealing the secrets of idiopathic pulmonary fibrosis. $N$ Engl J Med. 2019;380(1):94-96.

57. Wang Y, et al. Genetic defects in surfactant protein $\mathrm{A} 2$ are associated with pulmonary fibrosis and lung cancer. Am J Hum Genet. 2009;84(1):52-59.

58. Wert SE, Whitsett JA, Nogee LM. Genetic disorders of surfactant dysfunction. Pediatr Dev Pathol. 2009;12(4):253-274.

59. Nathan N, et al. Germline SFTPA1 mutation in familial idiopathic interstitial pneumonia and lung cancer. Hum Mol Genet. 2016;25(8):1457-1467.

60. Maitra M, Wang Y, Gerard RD, Mendelson CR, Garcia CK. Surfactant protein A2 mutations associated with pulmonary fibrosis lead to protein instability and endoplasmic reticulum stress. J Biol Chem. 2010;285(29):22103-22113.

61. Takezaki A, et al. A homozygous SFTPA1 muta- 
tion drives necroptosis of type II alveolar epithelial cells in patients with idiopathic pulmonary fibrosis. J Exp Med. 2019;216(12):2724-2735.

62. Lee JM, et al. Involvement of alveolar epithelial cell necroptosis in idiopathic pulmonary fibrosis pathogenesis. Am J Respir Cell Mol Biol. 2018;59(2):215-224.

63. Raghu G, Weycker D, Edelsberg J, Bradford WZ, Oster G. Incidence and prevalence of idiopathic pulmonary fibrosis. Am J Respir Crit Care Med. 2006;174(7):810-816.

64. Torres-González E, et al. Role of endoplasmic reticulum stress in age-related susceptibility to lung fibrosis. Am J Respir Cell Mol Biol. 2012;46(6):748-756.

65. Kamp DW, et al. Asbestos-induced alveolar epithelial cell apoptosis. The role of endoplasmic reticulum stress response. Am J Respir Cell Mol Biol. 2013;49(6):892-901.

66. Laing S, et al. Airborne particulate matter selectively activates endoplasmic reticulum stress response in the lung and liver tissues. Am J Physiol Cell Physiol. 2010;299(4):C736-C749.

67. Aggarwal S, et al. Heme scavenging reduces pulmonary endoplasmic reticulum stress, fibrosis, and emphysema. JCI Insight. 2018;3(21):120694.

68. Rooney SA. Chapter 7: Regulation of surfactant secretion. In: Rooney SA, ed. Lung Surfactant: Cellular and Molecular Mechanisms. R.G. Landes; 1998:29-45.

69. Vicary GW, Vergne Y, Santiago-Cornier A, Young LR, Roman J. Pulmonary fibrosis in Hermansky-Pudlak syndrome. Ann Am Thorac Soc. 2016;13(10):1839-1846.

70. Kook S, et al. Gene-edited MLE-15 cells as a model for the Hermansky-Pudlak syndromes. Am J Respir Cell Mol Biol. 2018;58(5):566-574.

71. Strikoudis A, et al. Modeling of fibrotic lung disease using 3D organoids derived from human pluripotent stem cells. Cell Rep. 2019;27(12):3709-3723.e5.

72. Korogi Y, et al. In vitro disease modeling of Hermansky-Pudlak syndrome type 2 using human induced pluripotent stem cell-derived alveolar organoids. Stem Cell Reports. 2019;12(3):431-440.

73. Young LR, et al. Epithelial-macrophage interactions determine pulmonary fibrosis susceptibility in Hermansky-Pudlak syndrome. JCI Insight. 2016;1(17):e88947.

74. Ng B, et al. Interleukin-11 is a therapeutic target in idiopathic pulmonary fibrosis. Sci Transl Med . 2019;11(511):eaaw1237.

75. Cook SA, Schafer S. Hiding in plain sight: interleukin-11 emerges as a master regulator of fibrosis, tissue integrity, and stromal inflammation. Annu Rev Med. 2020;71:263-276.

76. Summer R, et al. Matrix metalloproteinase activity in the lung is increased in Hermansky-Pudlak syndrome. Orphanet J Rare Dis. 2019;14(1):162.

77. Ban N, et al. ABCA3 as a lipid transporter in pulmonary surfactant biogenesis. J Biol Chem. 2007;282(13):9628-9634.

78. Cheong N, et al. ABCA3 is critical for lamellar body biogenesis in vivo. J Biol Chem. 2007;282(33):23811-23817.

79. Bullard JE, Nogee LM. Heterozygosity for ABCA3 mutations modifies the severity of lung disease associated with a surfactant pro- tein C gene (SFTPC) mutation. Pediatr Res. 2007;62(2):176-179.

80. Shulenin S, Nogee LM, Annilo T, Wert SE, Whitsett JA, Dean M. ABCA3 gene mutations in newborns with fatal surfactant deficiency. $N$ Engl JMed.2004;350(13):1296-1303.

81. Besnard V, et al. Conditional deletion of Abca 3 in alveolar type II cells alters surfactant homeostasis in newborn and adult mice. Am J Physiol Lung Cell Mol Physiol. 2010;298(5):L646-L659.

82. Rindler TN, et al. Alveolar injury and regeneration following deletion of ABCA3. JCI Insight. 2017;2(24):97381

83. Beers MF, et al. Aberrant lung remodeling in a mouse model of surfactant dysregulation induced by modulation of the Abca3 gene. Ann Anat. 2017;210:135-146.

84. Reyfman PA, et al. Single-cell transcriptomic analysis of human lung provides insights into the pathobiology of pulmonary fibrosis. Am J Respir Crit Care Med. 2019;199(12):1517-1536.

85. Zank DC, Bueno M, Mora AL, Rojas M. Idiopathic pulmonary fibrosis: aging, mitochondrial dysfunction, and cellular bioenergetics. Front Med (Lausanne). 2018;5:10.

86. Bratic A, Larsson NG. The role of mitochondria in aging. JClin Invest. 2013;123(3):951-957.

87. Bueno $\mathrm{M}$, et al. ATF3 represses PINK1 gene transcription in lung epithelial cells to control mitochondrial homeostasis. Aging Cell. 2018;17(2):e12720.

88. Jablonski RP, et al. SIRT3 deficiency promotes lung fibrosis by augmenting alveolar epithelial cell mitochondrial DNA damage and apoptosis. FASEB J. 2017;31(6):2520-2532.

89. Bueno M, et al. PINK1 attenuates mtDNA release in alveolar epithelial cells and TLR9 mediated profibrotic responses. PLoS One. 2019;14(6):e0218003.

90. Yu G, et al. Thyroid hormone inhibits lung fibrosis in mice by improving epithelial mitochondrial function. Nat Med. 2018;24(1):39-49.

91. Oldham JM, et al. Thyroid disease is prevalent and predicts survival in patients with idiopathic pulmonary fibrosis. Chest. 2015;148(3):692-700.

92. Kirkland JL, Tchkonia T. Cellular senescence: a translational perspective. EBioMedicine. 2017;21:21-28.

93. Newton CA, et al. Telomere-related lung fibrosis is diagnostically heterogeneous but uniformly progressive. Eur Respir J. 2016;48(6):1710-1720.

94. Dressen A, et al. Analysis of protein-altering variants in telomerase genes and their association with MUC5B common variant status in patients with idiopathic pulmonary fibrosis: a candidate gene sequencing study. Lancet Respir Med. 2018;6(8):603-614

95. Courtwright AM, El-Chemaly S. Telomeres in interstitial lung disease: the short and the long of it. Ann Am Thorac Soc. 2019;16(2):175-181.

96. Armanios MY, et al. Telomerase mutations in families with idiopathic pulmonary fibrosis. $N$ Engl J Med.2007;356(13):1317-1326.

97. Cogan JD, et al. Rare variants in RTEL1 are associated with familial interstitial pneumonia. Am J Respir Crit Care Med. 2015;191(6):646-655.

98. Degryse AL, et al. Telomerase deficiency does not alter bleomycin-induced fibrosis in mice. $\operatorname{Exp}$
Lung Res. 2012;38(3):124-134.

99. Liu T, et al. Telomerase activity is required for bleomycin-induced pulmonary fibrosis in mice. $J$ Clin Invest. 2007;117(12):3800-3809.

100.Povedano JM, Martinez P, Flores JM, Mulero F, Blasco MA. Mice with pulmonary fibrosis driven by telomere dysfunction. Cell Rep. 2015;12(2):286-299.

101. Naikawadi RP, et al. Telomere dysfunction in alveolar epithelial cells causes lung remodeling and fibrosis. JCI Insight. 2016;1(14):e86704.

102. Summer R, et al. Activation of the mTORC1/ PGC-1 axis promotes mitochondrial biogenesis and induces cellular senescence in the lung epithelium. Am J Physiol Lung Cell Mol Physiol. 2019;316(6):L1049-L1060.

103. Matos L, Gouveia AM, Almeida H. ER stress response in human cellular models of senescence. J Gerontol A Biol Sci Med Sci. 2015;70(8):924-935.

104. Kuwano K, et al. P21Waf1/Cip1/Sdi1 and p53 expression in association with DNA strand breaks in idiopathic pulmonary fibrosis. Am J Respir Crit Care Med. 1996;154(2 pt 1):477-483.

105. Schafer MJ, et al. Cellular senescence mediates fibrotic pulmonary disease. Nat Commun. 2017;8:14532.

106.Yao C, et al. Senescence of alveolar stem cells drives progressive pulmonary fibrosis Posted on bioRxiv October 28, 2019. https://doi.org/10.1101/820175.

107. Katzenstein AL, Myers JL. Idiopathic pulmonary fibrosis: clinical relevance of pathologic classification. Am J Respir Crit Care Med. 1998;157(4 pt 1):1301-1315.

108. Habermann AC, et al. Single-cell RNA sequencing reveals profibrotic roles of distinct epithelial and mesenchymal lineages in pulmonary fibrosis. Science Advances. 2020;6(28):eaba1972.

109. Strunz M, et al. Alveolar regeneration through a Krt8 ${ }^{+}$transitional stem cell state that persists in human lung fibrosis. Nat Commun. 2020;11(1):3559.

110. Kobayashi Y, et al. Persistence of a regeneration-associated, transitional alveolar epithelia cell state in pulmonary fibrosis. Nat Cell Biol. 2020;22(8):934-946.

111. Basil MC, Morrisey EE. Lung regeneration: a tale of mice and men. Semin Cell Dev Biol. 2020;100:88-100

112. Basil MC, et al. The cellular and physiological basis for lung repair and regeneration: past, present, and future. Cell Stem Cell. 2020;26(4):482-502.

113. Plantier L, et al. Ectopic respiratory epithelial cell differentiation in bronchiolised distal airspaces in idiopathic pulmonary fibrosis. Thorax. 2011;66(8):651-657.

114. Seibold MA, et al. A common MUC5B promoter polymorphism and pulmonary fibrosis. $\mathrm{N} \mathrm{EnglJ}$ Med.2011;364(16):1503-1512.

115. Schwartz DA. Idiopathic pulmonary fibrosis is a genetic disease involving mucus and the peripheral airways. Ann Am Thorac Soc. 2018;15(suppl 3):S192-S197.

116. Vaughan AE, et al. Lineage-negative progenitors mobilize to regenerate lung epithelium after major injury. Nature. 2015;517(7536):621-625.

117. Lee MG, Lee YH. A meta-analysis examining the 
association between the MUC5B rs35705950 $\mathrm{T} / \mathrm{G}$ polymorphism and susceptibility to idiopathic pulmonary fibrosis. Inflamm Res. 2015;64(6):463-470.

118. Mathai SK, et al. MUC5B variant is associated with visually and quantitatively detected preclinical pulmonary fibrosis. Thorax. 2019;74(12):1131-1139.

119. Chen $\mathrm{G}$, et al. XBP1S regulates MUC5B in a promoter variant-dependent pathway in idiopathic pulmonary fibrosis airway epithelia. Am J Respir Crit Care Med. 2019;200(2):220-234.

120. Hancock LA, et al. Muc5b overexpression causes mucociliary dysfunction and enhances lung fibrosis in mice. Nat Commun. 2018;9(1):5363.

121. Zuo W, et al. p63(+)Krt5(+) distal airway stem cells are essential for lung regeneration. Nature. 2015;517(7536):616-620.

122. Xi Y, et al. Local lung hypoxia determines epithelial fate decisions during alveolar regeneration. Nat Cell Biol. 2017;19(8):904-914.

123. Adams TS, et al. Single-cell RNA-seq reveals ectopic and aberrant lung-resident cell populations in idiopathic pulmonary fibrosis. Sci $A d v$. 2020;6(28):eaba1983.

124. Antoniades HN, Neville-Golden J, Galanopoulos T, Kradin RL, Valente AJ, Graves DT. Expression of monocyte chemoattractant protein 1 mRNA in human idiopathic pulmonary fibrosis. Proc Natl Acad Sci U S A. 1992;89(12):5371-5375.

125. Moore BB, et al. Protection from pulmonary fibrosis in the absence of CCR2 signaling. J Immunol. 2001;167(8):4368-4377.

126. Misharin AV, et al. Monocyte-derived alveolar macrophages drive lung fibrosis and persist in the lung over the life span.J Exp Med. 2017;214(8):2387-2404.

127. Venosa A, et al. Epithelial expression of an interstitial lung disease-associated mutation in surfactant protein- $\mathrm{C}$ modulates recruitment and activation of key myeloid cell populations in mice. J Immunol. 2019;202(9):2760-2771.

128. Young LR, et al. The alveolar epithelium determines susceptibility to lung fibrosis in Hermansky-Pudlak syndrome. Am J Respir Crit Care Med. 2012;186(10):1014-1024.

129. Raghu G, et al. CC-chemokine ligand 2 inhibition in idiopathic pulmonary fibrosis: a phase 2 trial of carlumab. Eur Respir J. 2015;46(6):1740-1750.

130. Johannson KA, et al. Acute exacerbation of idiopathic pulmonary fibrosis associated with air pollution exposure. Eur Respir J. 2014;43(4):1124-1131.

131. Wootton SC, et al. Viral infection in acute exacerbation of idiopathic pulmonary fibrosis. $\mathrm{Am} \mathrm{J}$ Respir Crit Care Med. 2011;183(12):1698-1702.

132. Lee JS, et al. Bronchoalveolar lavage pepsin in acute exacerbation of idiopathic pulmonary fibrosis. Eur Respir J. 2012;39(2):352-358.

133. Oda K, et al. Autopsy analyses in acute exacerbation of idiopathic pulmonary fibrosis. Respir Res. 2014;15:109.

134. Collard HR, et al. Acute exacerbation of idiopathic pulmonary fibrosis. An international working group report. Am J Respir Crit Care Med. 2016;194(3):265-275.

135. Song JW, Hong SB, Lim CM, Koh Y, Kim DS. Acute exacerbation of idiopathic pulmonary fibrosis: incidence, risk factors and outcome. Eur Respir J. 2011;37(2):356-363.

136. Collard HR, et al. Plasma biomarker profiles in acute exacerbation of idiopathic pulmonary fibrosis. Am J Physiol Lung Cell Mol Physiol. 2010;299(1):L3-L7.

137. Papiris SA, et al. High levels of IL- 6 and IL- 8 characterize early-on idiopathic pulmonary fibrosis acute exacerbations. Cytokine. 2018;102:168-172.

138. Miyazaki Y, et al. Higher serum CCL17 may be a promising predictor of acute exacerbations in chronic hypersensitivity pneumonitis. Respir Res. 2013;14:57.

139. Kulkarni AB, et al. Transforming growth factor beta 1 null mutation in mice causes excessive inflammatory response and early death. Proc Natl Acad Sci U S A. 1993;90(2):770-774.

140. Munger JS, et al. The integrin alpha $v$ beta 6 binds and activates latent TGF beta 1: a mechanism for regulating pulmonary inflammation and fibrosis. Cell.1999;96(3):319-328.

141. Coker RK, Laurent GJ, Jeffery PK, du Bois RM, Black CM, McAnulty RJ. Localisation of transforming growth factor beta 1 and beta $3 \mathrm{mRNA}$ transcripts in normal and fibrotic human lung. Thorax. 2001;56(7):549-556.

142. $\mathrm{Wu} \mathrm{H}$, et al. Progressive pulmonary fibrosis is caused by elevated mechanical tension on alveolar stem cells. Cell. 2020;180(1):107-121.e17.

143. Lee JH, et al. Anatomically and functionally distinct lung mesenchymal populations marked by Lgr5 and Lgr6. Cell. 2017;170(6):1149-1163.e12.

144.Zepp JA, et al. Distinct mesenchymal lineages and niches promote epithelial self-renewal and myofibrogenesis in the lung. Cell. 2017;170(6):1134-1148.e10.

145. Nogee LM, Dunbar AE, Wert S, Askin F, Hamvas A, Whitsett JA. Mutations in the surfactant protein $\mathrm{C}$ gene associated with interstitial lung disease. Chest. 2002;121(3 suppl):20S-21S.

146.van Moorsel CH, et al. SFTPA2 mutations in familial and sporadic idiopathic interstitial pneumonia. Am J Respir Crit Care Med. 2015;192(10):1249-1252.

147. Bullard JE, Wert SE, Whitsett JA, Dean M, Nogee
LM. ABCA3 mutations associated with pediatric interstitial lung disease. Am J Respir Crit Care Med. 2005;172(8):1026-1031.

148.Wambach JA, et al. Genotype-phenotype correlations for infants and children with ABCA3 deficiency. Am J Respir Crit Care Med. 2014;189(12):1538-1543.

149. Matsumura Y, Ban N, Ueda K, Inagaki N. Characterization and classification of ATP-binding cassette transporter $\mathrm{ABCA} 3$ mutants in fatal surfactant deficiency. J Biol Chem. 2006;281(45):34503-34514.

150. Brasch F, et al. Alteration of the pulmonary surfactant system in full-term infants with hereditary ABCA3 deficiency. Am J Respir Crit Care Med. 2006;174(5):571-580.

151. Tran I, Ji C, Ni I, Min T, Tang D, Vij N. Role of cigarette smoke-induced aggresome formation in chronic obstructive pulmonary disease-emphysema pathogenesis. Am J Respir Cell Mol Biol. 2015;53(2):159-173.

152. Kelsen SG, Duan X, Ji R, Perez O, Liu C, Merali S. Cigarette smoke induces an unfolded protein response in the human lung: a proteomic approach. Am J Respir Cell Mol Biol. 2008;38(5):541-550.

153. Malhotra D, et al. Heightened endoplasmic reticulum stress in the lungs of patients with chronic obstructive pulmonary disease: the role of Nrf2-regulated proteasomal activity. Am J Respir Crit Care Med. 2009;180(12):1196-1207.

154. Ather H, Zahid N. Recurrent renal cancer in BirtHogg-Dubé syndrome: a case report. Int J Surg Case Rep. 2018;42:75-78.

155. Goncharova EA, et al. Folliculin controls lung alveolar enlargement and epithelial cell survival through E-cadherin, LKB1, and AMPK. Cell Rep. 2014;7(2):412-423.

156. Kennedy JC, Khabibullin D, Boku Y, Shi W, Henske EP. New developments in the pathogenesis of pulmonary cysts in Birt-HoggDubé syndrome. Semin Respir Crit Care Med. 2020;41(2):247-255.

157. Sarkar S, et al. Impaired autophagy in the lipid-storage disorder Niemann-Pick type C1 disease. Cell Rep. 2013;5(5):1302-1315.

158. von Ranke FM, et al. Pulmonary involvement in Niemann-Pick disease: a state-of-the-art review. Lung. 2016;194(4):511-518.

159. Pacheco CD, Lieberman AP. Lipid trafficking defects increase Beclin-1 and activate autophagy in Niemann-Pick type C disease. Autophagy. 2007;3(5):487-489.

160. Liu T, et al. Telomerase and telomere length in pulmonary fibrosis. Am J Respir Cell Mol Biol. 2013;49(2):260-268. 